\title{
El proceso constituyente y la policía: El primer paso de un proyecto democratizador
}

\author{
The Constitutional Proccess and the Police: \\ The First Step in a Democratizing Proyect
}

\author{
Pascual Cortés Carrasco \\ GonZAlo García-CAMPO Almendros"
}

\begin{abstract}
Resumen
El presente artículo aborda el impacto que la discusión constituyente puede tener en la configuración institucional de la policía en Chile. Luego de una revisión de la regulación constitucional vigente, caracterizada por la alta autonomía de Carabineros de Chile (I), se examina, con carácter genealógico, qué tipo de poder se encuentra en la base de las instituciones policiales, con el objeto de mostrar que éstas presentan características intrínsecas que las diferencian de otros órganos de la administración del Estado (II). Con ambos antecedentes expuestos, se señala que la discusión constituyente puede sentar las bases de un proceso de democratización de la policía. El trabajo advierte, no obstante, que la democratización de la policía es un proyecto que excede la construcción de una nueva regulación, requiriendo así de una robusta participación ciudadana, tanto para que la actividad policial vaya efectivamente en el interés general de la ciudadanía (III.a), como para que las futuras reformas policiales tengan éxito, evitando algunos fracasos ocurridos en otros países del continente (III.b).
\end{abstract}

Palabras claves: Nueva Constitución; Autonomía Policial; Poder Policial; Participación ciudadana; Democratización de la policía.

\begin{abstract}
This article addresses the impact that the constituent discussion may have on the institutional arrangement of the police in Chile. After a review of the existing constitutional regulation, characterized by the high degree of autonomy granted to Carabineros de Chile (I), a genealogical examination of the kind of power that undergirds police institutions is presented, in order to show that theses display inherent characteristics that differentiate them from other organs of the State administration (II). With both antecedents outlined, it is argued that the constituent discussion can lay the groundwork for a process of democratization of the police. The article notices, nonetheless, that democratization of the police is an endeavor that exceeds the formulation of a new legal framework, thus requiring robust citizen participation, both in order for police action to be
\end{abstract}

Investigador independiente, Chile (pascualcortes@gmail.com). ORCID: http://orcid.org/0000-0003-2067-9105. Artículo recibido el 31 de mayo de 2021, y aceptado para su publicación el 16 de septiembre de 2021.

Universidad de Oxford, Reino Unido (ggarciacampo@gmail.com). ORCID: http://orcid.org/0000-0002-5588-0987. 
effectively oriented towards the general interest of citizens (III.a), and for future police reform to be successful, avoiding some of the failures that have occurred in other countries in the continent (III.b).

\section{Keywords: New Constitution; Police Autonomy; Police Power; Citizen Participation; Democratization of the police.}

\section{INTRODUCCIÓN}

Este trabajo pretende ofrecer respuesta a una pregunta fundamental: ¿qué impacto puede tener el proceso constituyente que -en el momento en que este número es publicado- vive Chile en la configuración institucional de la policía? La pregunta no solo tiene pertinencia académica. Desde hace al menos un lustro, la función policial ha sido objeto de severos cuestionamientos en Chile, ' debido a la ocurrencia de severos problemas de corrupción, ${ }^{2}$ al involucramiento de la policía en el homicidio de comuneros mapuche, ${ }^{3}$ y en la actuación de Carabineros durante el "estallido social”, que dejó, entre otras cosas, un reguero de mutilaciones oculares y lesiones graves. ${ }^{4} \mathrm{Si}$ todos los factores aquí listados dan pertinencia a la idea de que la policía necesita una reforma, el proceso constituyente ofrece una oportunidad para abordar aspectos esenciales de ella. Lo que este artículo pretende elucidar es de qué manera el proceso constituyente puede, efectivamente, sentar las bases de una transformación de las fuerzas policiales. Para ello, el artículo procede en tres pasos, representados en las respectivas secciones del trabajo.

En primer lugar, se revisa críticamente la configuración constitucional de la policía en Chile. Por medio de una revisión histórica, y de una sucinta mirada comparativa, se muestra que la regulación chilena favorece un alto grado de autonomía policial, que ha sido calificada como "autonomía político-institucional", por una parte, y "autonomía operativo-funcional”, por la otra." Esta autonomía, sostenemos, ha tenido un correlato en la inexistencia de una deliberación pública

${ }^{1}$ Como muestra, la aprobación a Carabineros -la principal fuerza policial del país- habría caído desde más de un $75 \%$ en 2017 a cerca de un 35\% en 2019, según mediciones de la misma encuesta.

En 2016 comenzó a ser investigado un desfalco al erario protagonizado por funcionarios de la institución que habría involucrado un monto cercano a los $\$ 35.000$ millones de pesos chilenos (aproximadamente 50 millones de dólares estadounidenses) y por la cual se habrían formalizado más de 130 investigaciones penales.

El 14 de noviembre de 2018, el comunero mapuche Camilo Catrillanca fue asesinado por funcionarios policiales apostados en la zona en el marco de una operación policial-militar informalmente denominada "Comando Jungla”, en virtud de la cual funcionarios policiales entrenados en Colombia, en la guerrilla contra las FARC, deberían contener las acciones políticas del pueblo mapuche. En principio, se habló de un enfrentamiento, versión que incluso fue avalada por el senador Felipe Kast, quien aseguró que "claramente el intercambio fue bastante duro". Tiempo después, el mismo parlamentario tuvo que desmentir sus declaraciones, asegurando en su cuenta de twitter que fue "engañado”.

${ }^{4}$ Según un artículo de especialistas médicos de la Unidad de Trauma Ocular del Hospital El Salvador, publicado en la revista científica $E y e$, de la editorial Nature, el número de traumas oculares producido por el uso de proyectiles de impacto cinético (conocidos como "perdigones antidisturbios") supone una proporción nunca vista en tan breve tiempo. Como ejemplo, los autores indican que "[l] a mayor cifra de trauma ocular se sitúa en un período de seis años, de 1987 a 1993, en el conflicto palestino-israelí. Ahí se registraron 154 casos. Nosotros registramos 182 casos en cerca de un mes y medio sólo en el Hospital del Salvador", según declararon en UNIVERSIDAD DE CHILE (2020): https:/www.uchile.cl/noticias/166739/nature-destaca-investigacion-sobre-dano-ocular-tras-estallido-social. Para el estudio completo, vid. RODRÍGUEZ et al. (2020).

CONTRERAS \& SALAZAR (2020a). 
respecto de los lineamientos básicos de la actividad policial en nuestro país. Por medio de este análisis crítico, la primera sección ofrece un diagnóstico del momento presente, que sirve para situar la discusión respecto de las perspectivas abiertas (y aquellas que no) por el proceso constituyente.

La segunda sección del artículo, sin embargo, no aborda directamente la respuesta a la pregunta central del artículo, sino que ofrece elementos cruciales para poder responderla. Una de las hipótesis centrales de este trabajo es que la posibilidad de reconducir la actividad policial al interés general, subordinando las instituciones policiales al poder civil, y asegurando su respeto por los elementos básicos de la democracia, supone reconocer el tipo de poder que las instituciones policiales ejercen. En esta sección buscamos mostrar que la policía (para el caso en análisis, Carabineros de Chile) despliega el así llamado "poder policial” del Estado, el cual debe ser comprendido como una forma de gobierno marcada por la arbitrariedad y la centralidad del concepto de orden. Esto tiene una importancia crucial, pues otorga a la policía rasgos que la distinguen de todas las otras instituciones que forman parte de la arquitectura del Estado. Así, aunque en una primera lectura la sección II parezca desconectada del argumento central, creemos que resulta esencial para una cabal comprensión de éste.

La tercera sección afronta directamente la respuesta a la pregunta central de este artículo. Para abordarla, considera los insumos provistos por las dos primeras secciones. Así, considerando, la especialidad de la regulación constitucional vigente, por una parte, y la especificidad del poder policial, por la otra, sostenemos que el proceso constituyente representa una oportunidad para iniciar un proceso de control democrático de las policías, que supone "desconstitucionalizar" los aspectos nucleares de su actividad y diseño institucional, para someterla a un mayor grado de control democrático. Esta “desconstitucionalización”, sin embargo, es solo un primer paso, ya que la entidad propia del poder policial requiere un control robusto de la ciudadanía para contrarrestar la arbitrariedad que le es inherente. En este sentido, esta sección llama a imaginar mecanismos de dirección y control sobre la actividad policial que vayan más allá de la regulación y de la acción de la judicatura, y que otorgue participación y protagonismo a la ciudadanía, especialmente a aquellos sectores que lidian cotidianamente con la policía. Estos mecanismos, se arguye, no solo son esenciales para remediar los déficits existentes en nuestro país, sino también para no repetir los errores recientes de otros países del subcontinente

\section{LA REGULACIÓN CONSTITUCIONAL Y LA AUTONOMÍA DE CARABINEROS DE CHILE}

Este trabajo tiene como punto de partida un diagnóstico que se desprende del tratamiento que la Constitución de 1980, actualmente vigente, otorga a la policía, en particular, a la institución de Carabineros de Chile. De acuerdo a dicho diagnóstico, el estatuto constitucional de la función policial desempeñada por Carabineros es uno que garantizaría una autonomía que viene dada, por un lado, por la sustracción de la actividad policial de la deliberación pública y, por otro, por la creación de un régimen de autonomía reforzada. El propósito de la presente sección es, entonces, ofrecer una reconstrucción general de una regulación constitucional de la policía que operaría como instrumento antidemocrático, en base a las dos constataciones recién señaladas. 


\subsection{Revisión histórica de la configuración legal de Carabineros de Chile}

Carabineros de Chile fue establecida como una institución policial, de carácter militar, el año 1927 a través del DFL № 2484 de 1927 del Ministerio del Interior (conocida como la primera "Ley Orgánica" de Carabineros), por iniciativa del militar Carlos Ibáñez en su calidad de comandante del Cuerpo de Carabineros del Ejército y ministro tanto de Guerra como de Interior. Carabineros de Chile surgió de un proceso de unificación de las policías fiscales, policías comunales y el Cuerpo de Carabineros del Ejército, con objetivos que incluían el aseguramiento del orden en ciudades y sectores rurales, la superación de la fragmentación y politización de las policías locales y el reforzamiento del principio de autoridad (considerandos $1^{\circ}$ a $5^{\circ}$ del DFL No 2484 de 1927). La nueva institución se estructuró como una policía centralizada y militarizada, tras décadas de modelamiento de la función policial "a imagen y semejanza de las Fuerzas Armadas, similar a países como España e Italia que poseen una larga tradición latifundista y de instituciones políticas tradicionales". ${ }^{6}$ Así, si hubiera que vincular a Carabineros de Chile a alguno de los tipos ideales de policía europea que se desarrollaron durante el siglo XIX, sin duda se acercaría al modelo de policía estatal militar, que se diferenciaría de las policías estatales civiles y municipales civiles. ${ }^{7}$ En 1960, el DFL № 213 del Ministerio de Hacienda (conocido como la segunda "Ley Orgánica” de Carabineros) reemplazó el decreto fundacional de Carabineros, desarrollando en mayor detalle sus funciones y ordenamiento interno, y atenuando su carácter militar al redirigir su dependencia desde el Ministerio de Defensa hacia el Ministerio del Interior. ${ }^{8}$

Carabineros de Chile fue una institución que no contó con regulación constitucional sino hasta el año $1971 .^{9}$ Su incorporación en la Constitución de 1925 ocurrió por medio de la Ley № 17.398, de 9 de enero de 1971, conocida como "Estatuto de Garantías Constitucionales", que modificó su artículo 22. Dicha regla -hasta entonces de muy exiguo contenido- fue complementada, pasando a precisar que la fuerza pública estaría "constituida única y exclusivamente por las Fuerzas Armadas y el Cuerpo de Carabineros, instituciones esencialmente profesionales, jerarquizadas, disciplinadas, obedientes y no deliberantes”. La misma norma agregaba que la dotación de estas instituciones solo podría fijarse por ley y que la incorporación a las mismas se realizaría a través de sus escuelas especializadas.

\footnotetext{
${ }^{6}$ Maldonado (1990), p. 3.

' Siguiendo a EMSLEY (1999), el modelo de policía estatal civil se vincularía al tipo victoriano de policía desarrollado en Londres a partir de 1829; el modelo de policía estatal militar se correspondería con los rasgos de la Gendarmerie nationale francesa, los Carabinieri italianos o el Royal Irish Constabulary, y el modelo de policía municipal es aquel que se desarrolló en distintos municipios y condados británicos o a nivel local en Francia, como el caso de los gardes champêtres. En el caso del modelo estatal militar, destinado especialmente a reafirmar la autoridad central del Estado, se dice que éste se habría desarrollado en Francia y, durante las invasiones napoleónicas, habría sido exportado a otras regiones de Europa continental como el norte y centro de Italia y las tierras de la Confederación del Rin y Prusia. Véase, sobre esto último EMSLEY (1999), p. 37.
}

${ }^{8}$ El carácter explícitamente militar no desapareció de la definición legal de Carabineros, sin embargo.

${ }^{9}$ La Constitución de 1925 establecía, en su texto original, anterior a la fundación de Carabineros de Chile, que "[l]a Fuerza Pública es esencialmente obediente. Ningún cuerpo armado puede deliberar” (art. 22). La Constitución de 1833 no hacía referencia a la policía como institución sino, en general, al poder de policía. Así, señalaba en su artículo 82 que “todos los objetos de policía y todos los establecimientos públicos están bajo la suprema inspección del Presidente de la República”. 
Con la fractura democrática provocada por el golpe cívico-militar de 1973 se inició un camino de remilitarización de Carabineros de Chile. ${ }^{10}$ Un primer antecedente del fortalecimiento de los rasgos militares y de la autonomía de Carabineros, que preocupó a la dictadura de Augusto Pinochet, puede encontrarse en el Decreto Ley № 444 de 27 de abril de 1974, del Ministerio del Interior. Esta norma tuvo tres propósitos: reafirmar el carácter técnico y militar de Carabineros, restableciendo su dependencia respecto del Ministerio de Defensa (art. $1^{\circ}$ ); ordenar la creación de una Subsecretaría de Carabineros (art. $2^{\circ}$ ); y ordenar la conformación de una Comisión Asesora para el desarrollo de un nuevo marco normativo para Carabineros (art. $3^{\circ}$ ), lo cual daría lugar, más adelante, al Decreto Ley № 1063 de 1975 (la tercera “Ley Orgánica” de Carabineros).

Consistentemente con este nuevo perfilamiento de Carabineros, la dictadura introdujo un modelo de regulación constitucional de la policía totalmente inédito. Una primera constatación, de carácter formal, se encuentra en la densidad de la regulación referida a las fuerzas armadas, de orden y seguridad pública, a la cual, por primera vez, se le dedicó un capítulo separado dentro del texto constitucional. Luego, en términos sustantivos, la Constitución contiene una serie de definiciones institucionales que están en la base de la afirmación formulada al inicio de este texto como diagnóstico, a saber, que la Constitución de 1980 se ocupó de sustraer la actividad policial de la deliberación pública, al mismo tiempo que dotó a Carabineros de Chile de una autonomía reforzada. Para algunos, este nuevo estatuto constitucional respondía a la intención de la junta militar de crear en la Constitución de 1980 un verdadero "poder de seguridad” que operaría como garante de la institucionalidad de la República y que debía resguardarse del control político de la autoridad democrática, lo que habría sido comunicado expresamente a la Comisión de Estudios para la Nueva Constitución a través del Mensaje del Ejecutivo de 10 de noviembre de 1977. ${ }^{11}$

\subsection{Principales rasgos de la regulación constitucional de Carabineros de Chile}

Las definiciones institucionales fundamentales que configuran el estatuto constitucional de Carabineros se encuentran en el actual Capítulo XI de la Constitución de 1980 (Capítulo X, en su versión original). Dentro de dicho capítulo, el artículo 101 inciso segundo define cuáles son las fuerzas de orden y seguridad (“Carabineros e Investigaciones”), consagra sus funciones ("dar eficacia al derecho, garantizar el orden público y la seguridad pública interior, en la forma que lo determinen sus respectivas leyes orgánicas") y fija su dependencia respecto del Ejecutivo a través del "Ministerio encargado de la Seguridad Pública”, lo que, al menos formalmente, hace de Carabineros una institución dependiente del Ejecutivo, a diferencia de órganos constitucionalmente autónomos que no tienen dependencia respecto de otros poderes del Estado. Se debe tener presente que, previo a la reforma constitucional de 2005 (Ley de Reforma Constitucional № 20.050), la Constitución establecía que las fuerzas militares y policiales existían "para la defensa de la patria, son esenciales para la seguridad nacional y garantizan el orden institucional de la República”, y establecía que dependerían del Ministerio de Defensa, lo que estaba en línea con las ya mencionadas definiciones ideológicas del régimen militar.

En cuanto a sus características, el mismo artículo 101 señala en su inciso tercero que las Fuerzas Armadas y Carabineros son cuerpos armados esencialmente obedientes, no deliberantes, profesionales, jerarquizados y disciplinados. En continuidad con la Constitución de 1925 (en su

\footnotetext{
${ }^{10}$ Maldonado (1990), p. 20.

${ }^{11}$ Contreras (2015), p. 320; Contreras \& Salazar (2020b), p. 15.
} 
versión reformada en 1971), el artículo 102 dispone que la incorporación a las plantas y dotaciones se realiza a través de las Escuelas propias de las fuerzas castrenses y de Carabineros. El artículo 104, por su parte, define un restringido sistema de nombramiento y remoción: el Presidente solo puede designar como Comandantes en Jefe o General Director de Carabineros a alguien entre las cinco mayores antigüedades de la oficialidad, el cual gozará de inamovilidad. Previo a la reforma de 2005, solamente era posible llamar a retiro anticipado a las cabezas de estas instituciones en casos calificados y con el acuerdo del Consejo de Seguridad Nacional. En la actualidad, dicha norma establece que el Presidente puede ocupar la figura del retiro anticipado mediante decreto fundado, informando previamente a ambas cámaras del Congreso Nacional. Finalmente, el artículo 105, que sufrió algunas reformulaciones con la Ley de Reforma Constitucional № 18.825 de 1989, reserva a una ley orgánica constitucional las normas básicas referidas a la carrera profesional, incorporación a plantas, previsión, antigüedad, mando, sucesión de mando y presupuesto de las Fuerzas Armadas y Carabineros.

Este modelo de regulación constitucional, y en especial el hecho de dedicar un capítulo separado y especial al tratamiento del poder militar y policial, ha sido calificado como anómalo y excepcional en el contexto comparado. ${ }^{12}$ Una revisión de distintos textos constitucionales pareciera confirmar lo anterior. Por ejemplo, al examinar las constituciones latinoamericanas actualmente vigentes se puede constatar que, de 18 textos constitucionales, ${ }^{13} 14$ contienen algún tipo de regulación constitucional de la policía, ya sea consagrando su existencia, definiendo su mandato general o bien perfilando su fisonomía institucional ${ }^{14}$. Dentro de dicho grupo, solamente 7 dedican un capítulo especial a las fuerzas armadas y policiales. ${ }^{15}$ La incorporación de la policía dentro del texto constitucional aparece como un fenómeno todavía más excepcional en el contexto de la Unión Europea. ${ }^{16}$ Solo 6 de los 27 países miembros regulan constitucionalmente la función policial, ${ }^{17}$ y de ellos, solamente 4 dedican un capítulo especial a la materia. ${ }^{18}$ Este análisis superficial no hace más

\footnotetext{
${ }^{12}$ Contreras \& Lovera (2020), p. 292.

${ }^{13}$ Las 18 constituciones revisadas, además de la chilena son Argentina (1994), Bolivia (2009), Brasil (1988), Colombia (1991), Costa Rica (1949), Cuba (2019), Ecuador (2008), El Salvador (1984), Guatemala (1985), Honduras (1982), México (1917), Nicaragua (1987), Panamá (1972), Paraguay (1992), Perú (1993), República Dominicana (2015), Uruguay (1967), Venezuela (1999).

${ }^{14}$ Correspondería a la lista de constituciones enumeradas en la nota anterior, excluyendo las de Argentina (1854), Cuba (2019), Guatemala (1985) y Uruguay (1967), las que no regulan a la policía.

${ }^{15}$ Se trata de las constituciones de Bolivia (Título VII, Capítulo II), Brasil (Título V, Capítulo III), Colombia (Capítulo 7), Ecuador (Capítulo 3, Sección 3), Panamá (Título XII), Paraguay (Capítulo V) y Venezuela (Título VII, Capítulo IV).

${ }^{16}$ Las constituciones revisadas corresponden a las de los 27 países de la Unión Europea: Austria (1920), Bélgica (1831), Bulgaria (1991), Croacia (1990), Chipre (1960), República Checa (1993), Dinamarca (1953), Estonia (1992), Finlandia (1999), Francia (1958), Alemania (1949), Grecia (1975), Hungría (2011), Irlanda (1937), Italia (1947), Letonia (1922), Lituania (1992), Luxemburgo (1868), Malta (1964), Países Bajos (1814), Polonia (1997), Portugal (1976), Rumania (1991), Eslovaquia (1992), Eslovenia (1991), España (1978) y Suiza (1999).

${ }^{17}$ Los textos constitucionales son los de Austria (1920), Bélgica (1831), Chipre (1960), Hungría (20122), Luxemburgo (1868) y Portugal (1976).

${ }^{18}$ Es el caso de las constituciones de Austria (Capítulo 3), Bélgica (Título VI), Chipre (Parte VIII) y Luxemburgo (Capítulo VII).
} 
que indicar que una regulación constitucional de la policía no es una tendencia extendida a nivel comparado.

A pesar de lo anterior, más interesante resulta observar que, entre aquellas constituciones que sí dedican un articulado a la policía, la reglamentación es más bien modesta, limitándose a enunciar su mandato y rasgos generales. Asimismo, entre las constituciones revisadas, la remisión a legislación de quórum supramayoritario aparece como una característica exclusiva del texto chileno. La tendencia comparada, prácticamente invariable, consiste en delegar a la legislación ordinaria la regulación de la organización y atribuciones específicas de la policía. La única y notable excepción es la constitución húngara de 2011 que, respecto de la policía, entrega a la "legislación cardinal” el tratamiento de los detalles de su organización y operación (art. 46 № 6 ), lo que supone reservar esta materia a un tipo de norma que requiere para su aprobación de dos tercios de los parlamentarios presentes. ${ }^{19}$ En principio, se podría pensar también que la constitución española realiza la misma operación, por cuanto señala, al referirse a las competencias de las comunidades autónomas, que lo relativo a la policía local es una materia de ley orgánica (art. 148 № 22). Sin embargo, dicha legislación exige un quórum de mayoría absoluta (art. 81), inferior a los cuatro séptimos de diputados y senadores en ejercicio que la constitución chilena de 1980 asigna a las leyes orgánicas constitucionales.

a. La autonomía de Carabineros como resultado de la regulación constitucional

De acuerdo al análisis hasta aquí presentado, es posible sostener que, al introducir una regulación de las fuerzas policiales, el texto constitucional de 1980 fue innovador, desde el punto de vista de la tradición constitucional nacional, y excepcional, en perspectiva comparada. Lo anterior permite profundizar el análisis y poner de relieve, como parte del diagnóstico aquí expuesto, que la Constitución de 1980 ha operado como instrumento antidemocrático debido a la magnitud de la autonomía concedida a la principal fuerza policial chilena, Carabineros de Chile. Esta autonomía puede ser analizada en dos niveles. Primero, por el lugar que se le ha dado a la policía en el esquema constitucional, lo que importa una decisiva exclusión de esta materia de la deliberación política. Y, segundo, por el régimen específico de autonomía que ha sido facilitado por el esquema constitucional revisado. Es conveniente referirse a cada uno de estos niveles de autonomía por separado.

En un primer nivel, existe una autonomía dada por el lugar específico que Carabineros ocupa en el esquema constitucional, lo que para algunos ha sido categorizado como "autonomía política institucional". ${ }^{20}$ Así, se ha conferido una esfera significativa de autonomía a Carabineros de Chile, que resguarda a la institución de los espacios de deliberación política, al otorgársele rango constitucional a las definiciones básicas de su diseño institucional y al reservar a una ley orgánica constitucional el desarrollo de cuestiones centrales del órgano policial. Ambas decisiones, como hemos visto, no guardan mayor correspondencia con diseños constitucionales en otras

\footnotetext{
${ }^{19}$ No deja de ser llamativo que la constitución vigente de Hungría fuera adoptada en 2011 bajo el gobierno del partido nacionalista y conservador Fidesz. Los(as) parlamentarios(as) de Fidesz fueron los únicos que concurrieron con su voto para la aprobación del texto constitucional, puesto que dicha colectividad contaba con dos tercios del parlamento. La constitución de 2011 fue adoptada a pesar de fuertes críticas desde la Unión Europea y Naciones Unidas debido a sus marcados tintes iliberales.

${ }^{20}$ CONTreras \& Salazar (2020b), p. 16.
} 
jurisdicciones. El hecho de que los principales contornos de la institucionalidad policial estén delineados en el capítulo XI de la Constitución tiene una implicancia obvia: cualquier alteración de aquello que ha quedado definido en el texto constitucional requerirá del voto de dos tercios de los diputados y senadores en ejercicio, porque operaría aquí el quórum reforzado de reforma constitucional del artículo 127 inciso segundo del texto vigente. Junto con esto, como ocurre con otras materias fundamentales del modelo que impuso la Constitución de 1980, la reserva de ley orgánica constitucional, y el control preventivo obligatorio del Tribunal Constitucional para este tipo de legislación, hacen de los rasgos basales de la institucionalidad policial un área prácticamente vedada a los procesos de deliberación democrática. Lo anterior, por cierto, no considera otros mecanismos del texto original, como el sistema binominal y la existencia de senadores designados ex uniformados, que reforzaban todavía más un blindaje normativo de las fuerzas militares y policiales según el cual cualquier reforma significativa del diseño de dichas instituciones debía contar con la anuencia de la derecha política, cuyos representantes en el parlamento, en ese período, habían tenido, en su mayoría, una relación cercana con la dictadura, cuando no habían sido derechamente parte de los equipos de la misma. ${ }^{21}$

En un segundo nivel, existe una autonomía que se verifica en diversos atributos de Carabineros de Chile, lo que ha sido denominado "autonomía operativa funcional". ${ }^{22}$ Varios de estos aspectos se encuentran en la Ley Orgánica Constitucional de Carabineros vigente, dictada en los últimos días del régimen dictatorial (se trata de la Ley № 18.961 Orgánica Constitucional de Carabineros de 7 de marzo de 1990, en adelante "LOC de Carabineros"). Contreras y Salazar ${ }^{23}$ identifican varios de ellos, entre los cuales se pueden destacar: las amplias atribuciones otorgadas al general director para decidir sobre cuestiones de carrera profesional, nombramientos, regímenes de calificación, ascensos y retiros; la regulación de un sistema diferenciado de previsión y seguridad social; el aseguramiento de recursos presupuestarios y extrapresupuestarios específicos; excepciones al régimen general de transparencia en virtud de su carácter militar, lo que se concretiza en la existencia de gastos de rendición reservada o secreta y la aplicación de la causal de secreto del artículo 436 del Código de Justicia Militar; ${ }^{24}$ la aplicación del Código de Justicia Militar, sin perjuicio de la reciente reducción de la jurisdicción militar introducida por las leyes № 20.477 y № 20.968; y, la existencia de un régimen administrativo disciplinario autónomo. Se puede sumar a este listado la capacidad de Carabineros de autorregularse en materias especialmente sensibles, como lo es la regulación de sus actuaciones en el mantenimiento del orden público y la utilización de armamento menos letal. ${ }^{25}$

\footnotetext{
${ }^{21}$ CONTRERas \& Salazar (2020a), p. 243.

${ }^{22}$ CONTRERas \& Salazar (2020b), p. 17.

${ }^{23}$ CONTReras \& Salazar (2020b), pp. 17-19.

${ }^{24} \mathrm{El}$ artículo 436 del Código de Justicia Militar lista qué documentos deben entenderse como secretos en el caso de las Fuerzas Armadas y Carabineros, incluyendo "los relativos a plantas y dotaciones” (№ 1); los "atinentes a planos o instalaciones de recintos militares o policiales y los planes de operación o de servicio de dichas instituciones” (№ 2); y "los concernientes a armas de fuego, partes y piezas de las mismas, municiones, explosivos, sustancias químicas” (№ 3), entre otros.

${ }^{25}$ Un ejemplo reciente lo constituye una modificación a los protocolos para el mantenimiento del orden público, en específico, el uso de la escopeta antidisturbios, armamento que ocasionó cientos de lesiones oculares entre manifestantes durante la crisis social de octubre de 2019. Dicha modificación, realizada por medio de la Orden general № 2780 de 14 de julio de 2020, reconoció como principales normas habilitantes "[l]as atribuciones conferidas al General Director
} 
Un grado de autonomía como el descrito puede ser calificado de deficitario, tanto porque el texto de la Constitución indica una cuestión totalmente distinta (Carabineros es una fuerza obediente y no deliberante), como por el inmenso poder del que está dotada la policía, que constituye, como desarrollaremos en la siguiente sección, el más importante repositorio institucional de la violencia legítima. Ambos factores aconsejan la existencia de un mayor grado de control sobre la policía, que permita a la comunidad política decidir de qué forma y con qué fines se ejercerá el monopolio de la fuerza materializado en la policía. La pregunta, por tanto, es cómo lograr este grado de control. La tercera sección de este artículo mostrará que el elemento fundamental es la participación ciudadana sobre la actividad policial, controlando y direccionando ésta. Sin embargo, la segunda sección resulta clave para fundamentar por qué esta participación es ineludible. La respuesta, sostendremos, se encuentra en el tipo de poder particular que la policía representa, y que la diferencia de todo otro órgano de la administración del estado. Para una mejor comprensión de este poder, un análisis de carácter genealógico es especialmente útil.

\section{LA INSTITUCIÓN POLICIAL COMO MANIFESTACIÓN DEL PODER POLICIAL}

\subsection{Origen y evolución del poder policial}

En su influyente trabajo de reconstrucción histórico-legal, Markus D. Dubber ha ligado los orígenes del "poder policial" a una forma de gobierno propia de la Grecia antigua. Según Dubber, el poder policial correspondía a la capacidad de mando patriarcal que el "jefe del hogar” ejercía sobre la unidad doméstica. El objetivo de este poder era mantener la felicidad y armonía de esta unidad, en los términos que el mismo jefe de hogar definiera. En esta forma de gobernanza no había, por tanto, consideraciones de dignidad o derechos individuales. Los medios que tenía el jefe de hogar para ejercer este poder eran prácticamente ilimitados. Así, el poder policial, entendido como una forma de dirección impuesto sobre una unidad doméstica, se oponía -y, en el caso del jefe de hogar, se complementaba- a la forma en que eran gobernadas las relaciones públicas en la polis, en las cuales el instrumento de gobierno era el derecho, estructurado en torno a consideraciones de igualdad y, con ellas, reglas de deliberación y toma de decisiones. En este esquema, el poder policial y el derecho aparecen como formas opuestas de regular las relaciones sociales, que sin embargo coexisten para permitir el decurso de la vida social. Sus ámbitos de aplicación, como se colige de lo expuesto, eran completamente distintos: el derecho era el instrumento de regulación de la actividad pública; la policía, el poder que el jefe de hogar ejercía sobre los miembros de la unidad doméstica. ${ }^{26}$

Para Dubber, el poder policial como forma de gobierno fue acogido por la cultura jurídicopolítico romana, y desde ahí fue adoptado por el derecho medieval. A contar del siglo XVI, el concepto de "policía” comenzó a ser objeto de un profundo desarrollo teórico y práctico en los principados germánicos, donde tendría lugar el origen de la así llamada "ciencia de la policía”, la cual correspondería a aquella rama del conocimiento cuyo objeto era la maximización de la "felicidad y el bienestar" de la comunidad política entendida como un todo. ${ }^{27}$ De esta forma, el poder

de Carabineros en los artículos 51 y 52, letras h) y p), de la ley № 18.961 'Orgánica Constitucional de Carabineros de Chile’; y el artículo 19º del Reglamento de Documentación, № 22, de Carabineros de Chile.”

${ }^{26}$ DUBBER (2005).

${ }^{27}$ KNEMEYER (1980); ZEDNER (2006). 
policial aparecía como una forma de gobierno que concebía a sus destinatarios como súbditos subordinados a un proyecto común, no existiendo consideraciones de respeto individual sobre los cuales erigirse. La "ciencia de la policía” era una forma de conocimiento que iba en interés del soberano, esto es, del monarca, no de aquellos que eran los destinatarios finales de las acciones de gobierno. ${ }^{28}$ El término policía, así entendido, comprendía al menos tres acepciones: (i) las condiciones para que existiera orden en la comunidad, entendidas como prerrequisitos de un buen orden; (ii) todas las leyes y reglas cuyo objeto era el establecimiento y la mantención de un buen orden; y, (iii) de forma más restringida, las reglas cuyo objeto específico eran las materias de policía, es decir, aquellas que regulaban las conductas constitutivas de desorden. ${ }^{29}$ En otras palabras, el "poder de policía” comprendía todos aquellos aspectos necesarios para producir una comunidad ordenada, objetivo para el cual el soberano contaba con una gran variedad de instrumentos.

El surgimiento de los estados constitucionales modernos, al alero de la Revolución Americana en 1776 y la Revolución Francesa en 1789, implicó un desplazamiento radical en los fundamentos de la legitimidad del poder de los Estados. Desprovistos de la legitimidad teológica que sostenía a las monarquías, las repúblicas que abrieron la puerta al siglo XIX debían encontrar un fundamento racional y secular capaz de justificar el ejercicio del poder. La idea de soberanía popular, según la cual, en términos gruesos, el poder soberano descansaba en la comunidad política, y no ya en el gobernante, es quizá la heredera más destacada de esta nueva comprensión de lo político. La comunidad política pasaba a ser detentadora del poder constituyente, y era, así, capaz de reinventar una y otra vez el régimen de vida en común. ${ }^{30}$ Esto dio origen, adicionalmente, a una visión radicalmente distinta del rol del derecho, especialmente de lo que hoy se conoce como derecho público, ya que éste pasaba a ser el instrumento que fundamentaba las capacidades y los límites de todo ejercicio de poder. En tanto forma de regulación de la actividad de gobierno, el derecho dotaba a ésta de legitimidad, sometiéndola a fines, y basando su ejercicio en la consideración de la igualdad de toda persona. ¿Qué pasaba, entonces, con la forma de gobierno que hemos caracterizado bajo el rótulo "policía”? Una respuesta tentativa es asumir su desaparición, subsumida bajo el alcance del derecho. Sin embargo, una mirada más atenta a las estructuras de gobierno propias de la modernidad, sostiene Dubber, permite constatar la pervivencia del poder policial. $^{31}$

Siguiendo la lectura de Dubber, el poder policial persiste hasta el día de hoy en todas aquellas estructuras de gobierno cuyo poder se despliega para asegurar la existencia de una comunidad ordenada, eliminando todas las amenazas a dicho orden. El concepto de orden que se sostiene es contingente, esto es, su contenido es definido por el mismo agente encargado de asegurarla. Tanto la definición de qué corresponde a orden, como el modo de asegurarla, son prerrogativas, en esta interpretación, del Estado. Dado el carácter polifacético de los Estados modernos, la existencia del poder policial puede encontrarse dispersa en éstos. No sería titularidad de una agencia específica ni prerrogativa de una instancia particular. Todas aquellas facultades cuyo fin es asegurar un determinado orden pueden ser reconducidas al poder policial del Estado. Sin embargo, hay una agencia estatal donde la remembranza del poder policial pervive con mayor nitidez: la policía. Bajo

\footnotetext{
${ }^{28}$ Preferimos evitar, aquí, el uso de conceptos como "pueblo", por considerarlos un anacronismo en virtud del período histórico analizado.

${ }^{29}$ ZEDNER (2006), p. 82.

${ }^{30}$ BÖCKENFÖRDE (2017).

${ }^{31}$ DUbBer (2005).
} 
esta comprensión, la policía es la instancia estatal cuyo fin primordial es afirmar la pervivencia de un determinado orden, eliminando todas las amenazas al mismo. Tal como dijéramos más arriba, el contenido del orden es definido por las instancias que detentan el poder para hacerlo, dotándolo así de una radical contingencia.

\subsection{La policía como heredera del poder policial}

La comprensión de la policía como una agencia estatal comprometida con la preservación de un determinado orden nos permite definirla de una forma que desafía ciertas comprensiones tradicionales acerca su identidad. Así, por ejemplo, una visión de la policía como la institución a la que corresponde velar por la vigencia del estado de derecho, o como la instancia cuya misión es el control del crimen, es puesta en entredicho por una comprensión más amplia y menos determinada, que reposa en la noción de orden. La policía aparece, de esta forma, como el último repositorio institucional de la fuerza. Como ha señalado Egon Bittner, un rasgo definitorio de la modernidad es la consecución de la paz por medios pacíficos. A diferencia de otras épocas, en que el objetivo de la paz era perseguido por medios violentos -Bittner cita, como paradigmático, el ejemplo las guerras desplegadas por el imperio romano para buscar la paz-, la modernidad se caracterizaría por disponer de medios que evitan el uso de la fuerza, como serían la diplomacia, a nivel internacional, y los tribunales de justicia, a niveles nacionales. Sin embargo, afirma Bittner, la fuerza es un fenómeno que no puede ser suprimido, ${ }^{32}$ y en las sociedades modernas quedaría replegada a tres sitios. En primer lugar, la auto defensa; en segundo, las autorizaciones específicas para que ciertas personas, que desarrollan determinados roles institucionales, ejerzan la fuerza en caso de ser necesario, como sucedería en el caso de las y los trabajadores de recintos de salud mental, y las y los funcionarios de gendarmería. El tercer repositorio de la fuerza lo constituiría la policía. ${ }^{33}$

En tanto repositorio de la fuerza, la policía posee una característica que lo diferencia de los otros dos. Se trata de la única instancia en la cual la autorización del uso de la fuerza es genérica, o "esencialmente ilimitada", ${ }^{34}$ pues no existe una restricción a priori de los casos en que la policía puede desplegar la fuerza, no obstante existir límites en lo que podríamos llamar el grado de fuerza desplegable (de esta forma, cobra sentido el llamamiento al "uso mínimo de la fuerza" que se suele exigir a las y los agentes policiales). A partir de la descripción anterior, Bittner define a la policía como "nada más que el mecanismo para distribuir la fuerza situacionalmente justificada en la sociedad”. Esta definición, provista de amplitud, ofrece, sostiene Bittner, tres ventajas. En primer lugar, es concordante con las expectativas que la gente suele tener cuando acude a la policía. En segundo lugar, es capaz de dar cuenta de los poderes que se suelen atribuir a las y los funcionarios policiales. En tercer lugar, esta concepción de la policía permite dar una lectura coherente de la diversidad de actividades que la policía lleva a cabo en sociedades democráticas. ${ }^{35}$

La definición de la policía que más arriba hemos ofrecido tiene dos consecuencias importantes. En primer lugar, la policía es definida por una capacidad (el uso de la fuerza legítima

\footnotetext{
${ }^{32}$ Bittner afirma esto de manera descriptiva, para señalar aquellos sitios en que pervive la fuerza. No tiene un compromiso valorativo respecto de las razones por las cuales la fuerza existe, o respecto de una posible supresión de la misma.

${ }^{33}$ BITTNER (1970), pp. 36 y ss.

${ }^{34}$ BITTNER (1970), p. 37.

${ }^{35}$ BiTtNer (1970), p. 39.
} 
de forma prácticamente ilimitada) y no por una(s) determinada(s) función(es). En segundo lugar, el poder de la policía como institución tiene un componente irreductiblemente discrecional. Dado que el rango de acciones en que la fuerza legítima puede ser utilizada es indefinible a priori, su uso queda siempre sometido al juicio de quien debe desplegarla. El criterio para decidir acerca de la pertinencia de su utilización, a pesar de la vaguedad con que puede formularse, tiene que ver con la protección de un cierto estado de orden. Provista, así, de discreción y una considerable amplitud en sus criterios de decisión, la policía como institución puede ser leída a la luz del poder policial, en los términos en que lo hemos descrito en este artículo. Así, aparece como la institución diseñada para mantener un cierto orden, de contenido indeterminado y por medios diversos. Esta caracterización impacta en los modos en que el diseño institucional puede ser imaginado. Al ser una institución en cuya raíz se encuentra un poder cuyo control resulta inherentemente difícil y arbitrario, la policía debe estar diseñada de tal forma que ese poder pueda ser contenido y controlado en algún grado. Es posible afirmar, según lo revisado hasta este punto, que la regulación vigente produce el efecto contrario, al reforzar la autonomía de la institución. La discusión constituyente, por tanto, puede ser una oportunidad para imaginar un diseño institucional distinto. A esto se aboca la sección siguiente.

\section{EL PROCESO CONSTITUYENTE COMO OPORTUNIDAD PARA UNA POLICÍA DIFERENTE. MÁS ALLÁ DE LA REGULACIÓN}

\subsection{Desconstitucionalización de la regulación policial; nuevos arreglos institucionales}

El recorrido que hemos hecho en las dos primeras secciones nos permite comenzar a responder la pregunta central que guía este artículo: ¿qué puede ofrecer el proceso constituyente para imaginar una configuración distinta de la policía? Responder esta pregunta -es lo que hemos querido sostener- exige tomar en consideración tanto la especialísima configuración constitucional que ha tenido históricamente la policía en Chile como la naturaleza del poder policial que la policía representa. Dicho de otra forma, solo tomando en cuenta ambos elementos podremos responder adecuadamente qué lugar debe ocupar la policía en nuestros arreglos sociales. En referencia al primero de estos factores, el proceso constituyente es una oportunidad para eliminar de la Constitución, (y para quitar los quórums supra mayoritarios hoy vigentes), a la regulación sobre los aspectos fundamentales de la institución policial. Como vimos en la Sección I, la arquitectura constitucional ha dotado a Carabineros de Chile de un alto grado de autonomía "políticoinstitucional” y de autonomía "operativo-funcional”. Si bien la primera fue reducida con las reformas constitucionales del año 2005, la autonomía de carácter operativo-funcional sigue siendo muy alta, y permite a la propia institución tomar decisiones cruciales sobre sus actuaciones sin que exista una supervisión sustantiva de las autoridades civiles.

La arquitectura constitucional a que hemos hecho mención reposa, esencialmente, sobre dos cuerpos normativos, que hemos descrito sucintamente en la primera sección: el capítulo XI de la Constitución Política de la República y la Ley Orgánica Constitucional de Carabineros de Chile. Por medio del reconocimiento en estos instrumentos de algunos aspectos fundamentales del diseño y la actividad de Carabineros de Chile, se dificulta sobremanera a la ciudadanía -tanto de forma directa como por medio de sus representantes- tener alguna injerencia acerca del modo en que la actividad policial es llevada a cabo, debido a que lograr algún cambio en tales aspectos requiere de altísimos quórums parlamentarios. De esta forma, se asegura, en la práctica, la permanencia de ciertas definiciones básicas, que, adicionalmente, nunca fueron objeto de discusión democrática, debido al origen de la regulación. Dicho en forma tal vez levemente pretenciosa, pero contundente, 
la imposibilidad democrática opera no solo en forma sincrónica, es decir, de cara al presente, sino también diacrónica, prolongando una historia de déficit democrático. El proceso constituyente ofrece una oportunidad a este respecto porque permite someter aquellos aspectos fundamentales del diseño institucional y de la actividad policial al control de la ciudadanía, sujetándolos, por ejemplo, a procedimientos de reforma que no estén obstaculizados de antemano por quórums contra mayoritarios. ${ }^{36}$

Este proceso, que en términos gruesos podemos llamar de "desconstitucionalización", representa, sin embargo, solamente un primer paso para construir un diseño institucional capaz de ejercer un control y dirección efectivo sobre la policía. Una vez que los aspectos esenciales de su regulación son sometidos al escrutinio democrático, cabe preguntarse qué tipo de arreglos institucionales podrían facilitar dicho control y dirección. A pesar de que una respuesta cabal y exhaustiva excede los límites de este trabajo, en lo que resta de esta sección queremos ofrecer algunas orientaciones preliminares, cuya generalidad pretende hacerse cargo de que uno de los objetivos más importantes de la desconstitucionalización es la democratización de las decisiones respecto de la policía, por lo cual queremos ofrecer algunos lineamientos gruesos que sean compatibles con la diversidad de posibilidades que una discusión abierta puede arrojar. En términos generales, se debe permitir el control de la ciudadanía sobre la policía. Esto exige discutir formas de injerencia sobre las decisiones policiales que permitan una participación efectiva de la ciudadanía, de tal forma que hagan más probable que la labor policial sea realizada en cumplimiento del interés general. Un paso así supone expandir la comprensión usual acerca de las formas utilizadas para controlar la actividad policial. Durante las últimas décadas, bajo la idea de que la policía debe "responder al derecho", se ha extendido un modelo de control de la actividad policial que recae en la judicatura. ${ }^{37}$ Por la extensión de este sentido común, creemos que los modelos que permiten la participación ciudadana tienen una inmediata resonancia contraintuitiva. Para hacernos cargo de ella, conviene responder en términos explícitos a la siguiente pregunta: ¿qué valores o bienes pueden ser favorecidos por medio de este tipo de arreglos institucionales? La respuesta puede cifrarse en los dos niveles expuestos a continuación.

En primer lugar, por medio de la participación en las decisiones acerca de la actividad policial se permite orientar dicha actividad hacia el cumplimiento del interés general. En términos gruesos, ese interés general puede ser entendido como la provisión de seguridad. Nuevamente, esto puede parecer extraño a primera vista, pues la seguridad se suele asociar con una actividad técnica especial, que requiere determinada experticia y que funciona, muchas veces, en secreto. Sin negar que muchos de estos aspectos pueden ser ciertos en ocasiones, creemos que la seguridad debe ser entendida como un bien público, en la cual el Estado tiene un rol indispensable que jugar. Así, el Estado tiene los instrumentos para permitir que el acceso a los medios que permitan a las personas experimentar su cotidianeidad de forma segura sean distribuidos de acuerdo a la condición de ciudadanía, y no se encuentren mediados por la capacidad de pago, que tiende, como es propio de los bienes provistos en el mercado, a una distribución desigual. ${ }^{38}$ Para que esto suceda, es fundamental que la ciudadanía tenga formas de participar (nuevamente, debemos recordarlo, ya sea

\footnotetext{
${ }^{36}$ CONTRERAS \& SALAZAR (2020b).

${ }^{37}$ REINER et al. (2019), p. 244.

${ }^{38}$ Sobre la seguridad como bien público, y el rol indispensable del Estado, véase LOADER \& WALKER (2001); LOADER \& WALKER (2007) y ZEDNER (2009).
} 
de manera directa o por medio de sus representantes) en las decisiones acerca del modo en que la actividad policial es desarrollada.

En segundo lugar, los arreglos institucionales capaces de desconcentrar el poder sobre la actividad policial pueden contribuir a un mayor control sobre el desempeño de una institución que representa, como hemos dicho, un poder inherentemente incontrolable. La aparente paradoja debe ser notada. La policía, en tanto institución, representa un poder que, como argüimos largamente en la sección II, reside en el Estado, y que no es controlado en las formas tradicionales diseñadas para limitar el poder, como suelen ser, típicamente, los derechos fundamentales, sino que obedece a nociones abstractas y generales, como el orden. Por esto, un mayor grado de desconcentración de la toma de decisión sobre la institución que encarna este poder, permite, sino contrarrestarlo de manera absoluta, sí tender a disminuir la arbitrariedad con que es utilizado. Es por esto que un control efectivo sobre la actividad policial no puede ser logrado solo mediante instrumentos legales, sino que requiere de la participación ciudadana. Esto nos lleva a relevar el rol que cabe a los grupos sociales que suelen convivir cotidianamente con una sobrepresencia policial. Como es de público conocimiento, durante al menos las dos últimas décadas, al alero de distintos programas, existen lugares que (denominados usualmente, por los instrumentos administrativos, como "barrios") han incorporado, como parte del paisaje cotidiano, una alta presencia policial. Valga como ejemplo el caso de la población La Legua, en la cual, según un informe emitido por el Instituto Nacional de Derechos Humanos, rotaban cotidianamente 72 funcionarios/as policiales, divididos en cuatro turnos de 18 funcionarios/as cada uno. ${ }^{39}$

Cuando esta sobrepresencia policial ${ }^{10}$ no va acompañada de una participación efectiva de la ciudadanía respecto de sus fines, y de los medios utilizados para cumplirlos, es probable que el poder de la policía devenga en abusos y arbitrariedades, pues es desplegado para cumplir con objetivos cuya concreción requiere, inevitablemente, de altísimos grados de discreción, tales como la mantención del orden o la seguridad. Lo que podemos ver en lugares como La Legua es que, efectivamente, los abusos policiales han sido frecuentes, ${ }^{41}$ al tiempo que los indicadores de violencia interpersonal no han disminuido durante el tiempo que los funcionarios policiales se han apostado permanentemente en el lugar, uno de los objetivos que, teóricamente, debía cumplir su presencia. ${ }^{42}$ Estos hechos, a nuestro juicio, no muestran una desviación de los fines para los cuales la policía es utilizada (aunque sí representen una desviación, incluso en ocasiones una desviación criminal, respecto de la legalidad imperante), sino lo que es probable que suceda cuando el poder policial es desplegado sin contrapesos. Los arreglos institucionales cuyos lineamientos generales sugerimos en esta sección buscan contrapesar este fenómeno, es decir, que exista algún tipo de control sobre el poder policial, de tal forma que su inherente arbitrariedad sea contenida. Por esto, adicionalmente, sostenemos que los grupos sobre-policiados deberían jugar un rol protagónico en las decisiones acerca de las formas en que el poder policial es utilizado. Dado que este poder es parte de su paisaje

\footnotetext{
${ }^{39}$ INDH (2015), p. 20.

${ }^{40}$ Utilizamos el término "sobre-presencia policial" de forma neutra en términos valorativos. Su uso obedece, más bien, a razones comparativas: se trata de una cantidad de funcionarios/as policiales muy superior a la que se suele encontrar en lugares de similares dimensiones físicas y demográficas.

"Comité de Defensa y Promoción de Derechos Humanos de la legua \& Universidad Diego Portales (2017) y CORTÉS \& GARCÍA-CAMPO (2018).

${ }^{42}$ Cámara de Diputados (2016).
} 
cotidiano, son ellos los más capacitados para favorecer que este poder contribuya a la provisión de seguridad, y no sea una permanente fuente de abusos. ${ }^{43}$

Una salvedad, sin embargo, debe ser hecha. Los arreglos institucionales desconcentradores de las decisiones acerca de la labor policial, que hemos bosquejado en el párrafo anterior, no deben ser confundidos con los modelos de "policía comunitaria", que han ocupado buena parte de las propuestas de reformas policiales a lo largo de los últimos años. ${ }^{4}$ Estos modelos insisten en la necesidad de comprender a la policía como proveedora de servicios, y a que ésta sea "cercana" a los lugares en que desarrolla su actividad diaria, conociendo las necesidades propias de los lugares que "atienden" (una palabra que se aviene con la comprensión de "provisión de servicios"). Sin embargo, el modelo de "policía comunitaria" suele obviar la pregunta por la distribución del poder en la relación que establecen las comunidades con los cuerpos policiales, abogando por la cercanía como el factor clave para producir resultados positivos. A nuestro juicio, esta omisión es crucial, y diferencia las propuestas que sostenemos en este artículo de los modelos de "policía comunitaria”, pues creemos que más allá de la cercanía y el conocimiento del entorno local (aspectos necesarios, sin duda), lo que se requiere es que las personas que a diario interactúan con la policía tengan un mayor control sobre el modo en que ésta despliega sus funciones, de tal forma de prevenir el uso arbitrario del poder y los abusos. ${ }^{45}$

\subsection{La necesidad de participación ciudadana en las reformas policiales}

Lo que hemos querido argumentar hasta aquí es que el proceso constituyente ofrece una oportunidad valiosa para "desconstitucionalizar" los aspectos fundamentales de la regulación policial, esto es, someterlos al escrutinio democrático que hoy se encuentra impedido por dicha regulación. Hemos señalado, sin embargo, que para diseñar una policía que verdaderamente desarrolle sus funciones en cumplimiento del interés general no basta con imaginar nuevos dispositivos de regulación legal, sino que se requiere una participación efectiva de la ciudadanía en el control de la actividad policial. Solo esta participación, hemos insistido, permite ejercer algún tipo de dirección sobre el poder que detenta la institución policial. El control de la policía, dicho en forma sintética, requiere una robusta participación de la ciudadanía. Sin embargo, en lo que resta de esta sección queremos destacar que la participación ciudadana en el devenir de las reformas policiales no son solamente una condición para poder controlar a la policía en el día a día, sino un requisito esencial para que cualquier reforma policial tenga éxito. Dicho de modo inverso, creemos que cualquier reforma que se intente llevar a cabo sin que exista una discusión política sobre la función de la policía, no tiene gran perspectiva de futuro.

Este punto ha sido advertido desde los estudios policiales, sosteniéndose que las prácticas policiales no son particularmente reactivas a los cambios formales en la regulación legal, por lo que cualquier cambio significativo supone una modificación del rol social estructural de la policía. ${ }^{46}$

\footnotetext{
${ }^{43}$ Simonson (2021).

${ }^{4}$ DAMMERT (2019).

${ }^{45}$ Para una crítica similar a los modelos de policía comunitaria en el caso estadounidense, véase SKLANSKY (2005), pp. 1810-1814.

${ }^{46}$ Reiner analiza los cambios concretos tras la Police and Criminal Evidence Act (PACE) de 1984 en Inglaterra y Gales y sostiene que, a pesar de cambios significativos, la reforma no alcanzó importantes áreas de la práctica policial, porque
} 
Asimismo, la ausencia de un alineamiento político sustantivo sobre la función del poder policial puede explicar, al menos en parte, el fracaso de múltiples intentos de reforma policial en América Latina. Como bien ha observado Yanilda González, la policía detenta una capacidad extraordinaria para bloquear o incluso desmantelar esfuerzos de reforma. ${ }^{47}$ Para González, las reformas menores, enfocadas en un nivel operativo, muchas veces descuidan un contexto político e institucional más amplio que otorga un considerable poder estructural a la policía para defender sus prerrogativas y frustrar reformas. ${ }^{48}$ Yanilda González también ha sostenido, en base a un estudio de los casos de Argentina, Brasil y Colombia, que en escenarios políticos con preferencias fragmentadas, una reforma policial aparecerá como poco conveniente en términos electorales, situación que solo puede superarse en la medida en que se produzca una convergencia de posiciones políticas capaz de contrapesar el poder de la policía. ${ }^{49}$ Una discusión propiamente constituyente sobre el poder policial y la policía puede favorecer ese tipo de convergencia.

En Chile, desde fines de 2019 hasta hoy, distintas propuestas de reforma policial han sido formuladas por comisiones convocadas por el gobierno y el Congreso, así como por centros de pensamiento. La creación de un Ministerio de Seguridad Pública que, entre otras funciones, se encargue de coordinar a las policías y ejercer un rol de monitoreo externo; una mejora de los estándares de transparencia, la responsabilización y la rendición de cuentas; cambios en los criterios de ingreso, promoción y retiro de la carrera policial; adecuaciones en los procesos de formación; cambios para garantizar la equidad de género; avances en la especialización funcional; y, la creación de una oficina independiente de control de la conducta policial, son algunas de ellas. ${ }^{50}$ La mayoría de esas propuestas son deseables y pueden contribuir a una democratización de Carabineros de Chile. Sin embargo, su impacto será limitado si no se presentan como la consecuencia de una discusión ciudadana previa. La deliberación constituyente podrá concluir en la desconstitucionalización de las reglas básicas del poder policial y Carabineros de Chile -lo cual, se ha dicho, podría favorecer la reducción de su amplísima autonomía- o bien podrá consagrar nuevas reglas, distintas a las del texto de 1980. Con independencia del resultado, el último argumento aquí desarrollado sostiene que aquello que no puede ser soslayado es el debate sustantivo sobre la función que se le otorgará a la policía.

\section{CONCLUSIONES}

Este trabajo comienza sosteniendo, a modo de diagnóstico, que la Constitución de 1980 consagró un régimen que, por una parte, ha sustraído a la actividad policial de la deliberación pública, y por otra, creó una esfera de autonomía reforzada para Carabineros de Chile. Este diseño puede ser caracterizado como una ruptura respecto de la tradición constitucional chilena y, además, inusual en perspectiva comparada. Un diagnóstico como el ofrecido -compartido por parte de la

los cambios reales dependen de la cultura policial y ésta, a su vez, depende del rol socialmente determinado de la policía ["the structurally determined social role of the police"]. Véase REINER et al. (2019), p. 241.

${ }^{47}$ GONZÁlez (2019a), p. 291.

${ }^{48}$ GONZÁLEZ (2019a), p. 293.

${ }^{19}$ GONZÁLEZ (2019b).

${ }^{50}$ Véase Consejo de Reforma a Carabineros (2020), Comisión de Reforma a Carabineros (2019) y Duce \& DAMMERT (2019). 
doctrina constitucional chilena - llamaría a imaginar un diseño constitucional alternativo en el proceso constituyente en ciernes. Preliminarmente, la prioridad pareciera encontrarse en la necesidad de desmontar un arreglo constitucional que ha imposibilitado una discusión democrática sobre la policía.

Se ha intentado contribuir a la discusión constitucional sosteniendo en la sección II que la policía, como institución, es la principal heredera de un tipo especial de poder que la convierte en un órgano de la burocracia estatal difícilmente asimilable a otras entidades públicas. Tener en cuenta este antecedente es relevante para entender las limitaciones que tiene el derecho como herramienta de control y dirección de la policía. Se ha sugerido, en consecuencia, que, si bien la desconstitucionalización de las reglas fundamentales de Carabineros de Chile aparece como un primer paso para permitir una reforma democrática, el paso siguiente debiese tener especialmente en consideración la importancia de promover una discusión pública y ciudadana sobre los fines que socialmente se le asignan a la policía. Dicha conversación debiese ser capaz de visibilizar también la vOz de los grupos que han sido objeto preferente de intervención policial. Rodear a la institucionalidad policial de un proceso de deliberación ciudadana es, se sostiene aquí, una vía más promisoria para controlar a la policía, que la mera confianza en las herramientas tradicionales del estado de derecho. Junto con esto, se ha argumentado que la discusión política acerca del rol de la policía es indispensable para que cualquier reforma policial futura tenga alguna perspectiva de éxito. Distintas experiencias sugerirían que las reformas que no van precedidas de una discusión sobre la función de la policía están destinadas a tener un efecto limitado. En suma, el proceso constituyente tiene la potencialidad de permitir una participación ciudadana sobre las definiciones fundamentales de la función policial que resulta crucial para controlarla y reformarla. Hasta hoy, bajo el esquema constitucional vigente, estas cuestiones parecen imposibles. 


\section{Bibliografía CitADA}

BiTTner, Egon (1970). The functions of the Police in Modern Society (National Institute of Mental Health).

BÖCKENFÖRDE, Ernst Wolfgang (2017). Constitutional and political theory: Selected writings (Vol. 1) (Oxford University Press).

CÁmARA DE Diputados (2016). Informe de la comisión especial investigadora encargada de recabar antecedentes sobre los actos del gobierno vinculados a intervenciones policiales y sociales en barrios críticos entre los años 2001 y 2015.

Comisión de Reforma a CARAbineros (2019): "Propuesta de reforma a Carabineros de Chile", en: $\quad$ https://derecho.udp.cl/wp-content/uploads/2020/01/20200129 Propuesta-de-laComisio\%CC\%81n-de-Reforma-a-Carabineros-VFF-1.pdf

Comité de Defensa y Promoción de Derechos Humanos de la Legua y Universidad Diego Portales (2017). A quince años de intervención estatal. Violencia policial en La Legua (Universidad Diego Portales).

Consejo de Reforma a Carabineros (2020): "Informe del Consejo de Reforma a Carabineros", en: $\quad$ https://cdn.digital.gob.cl/filer_public/52/fe/52fe7434-e81b-48b9-b31479cd56ce134f/consejo_carabineros_v2.pdf

Contreras, Pablo (2015). “Las Fuerzas Armadas en la Constitución”, en Bassa, Jaime, Ferrada, Juan Carlos y Viera, Christian (eds.), La Constitución chilena. Una revisión crítica a su práctica política (LOM), pp. 315-338.

Contreras, Pablo y Lovera, Domingo (2020). La Constitución de Chile (Tirant Lo Blanch).

Contreras, Pablo y Salazar, Sebastián (2020a). “'Obedientes y No Deliberantes': Fuerzas Armadas, Autonomía y Control Democrático en Chile”, Revista Ius et Praxis, Año 26, № 2, pp. 232-253.

Contreras, Pablo y Salazar, Sebastián (2020b). "Desconstitucionalizar para democratizar: Las Fuerzas Armadas y las Policías en la Nueva Constitución”, Política - Revista de Ciencia Política, Vol. 58, № 1, pp. 11-34.

CORTÉs, Pascual y García-CAmpo, Gonzalo (2018). "Violencia y déficits institucionales en la intervención estatal en la población La Legua”, Revista CIS, Vol. 24, pp. 63-77.

DAmmerT, Lucía (2019). “Challenges of Police Reform in Latin America” en SIEDER, Rachel; Ansolabehere, Karina y Alfonso, Tatiana (eds.), Routledge Handbook of Law and Society in Latin America (Routledge).

DubBer, Markus (2005). The police power: Patriarchy and the foundations of American government (Columbia University Press).

Duce, Mauricio y DAMmerT, Lucía (2019): "Propuestas para iniciar un proceso de reforma a Carabineros de Chile”, Fundación Espacio Público, en: https://www.espaciopublico.cl/wpcontent/uploads/2019/11/Propuestas-para-iniciar-proceso-de-reformas-a-Carabineros-deChile.pdf 
Emsley, Clive (1999). “A typology of nineteenth-century police”, Crime, History \& Societies, Vol. $3, \mathrm{~N}^{\circ} 1$, pp. 29-44.

GonzÁlez, Yanilda (2019a). "The Swinging Pendulum of Police Reform in the Americas”, Current History, November 2019, pp. 291-297.

GonZÁLEZ, Yanilda (2019b). "The social origins of institutional weakness and change. Preferences, power, and police reform in Latin America”, World Politics, Vol. 71, № 1 (January 2019), pp. 44-87.

Instituto Nacional de Derechos Humanos (INDH) (2015). Estudio de Casos. Violencias y Derechos Humanos en La Legua.

Knemeyer, F.L. (1980). "Polizei”, Economy and Society, Vol. 9, № 2, pp. 172-196.

LoAder, Ian, y Walker, Neil (2001). "Policing as a Public Good: Reconstituting the Connections between Policing and the State”, Theoretical Criminology, Vol. 5, № 1, pp. 9-35.

LOAder, Ian y Walker, NeIL (2007). Civilizing security (Cambridge University Press).

Maldonado, Carlos (1990). "Los Carabineros de Chile. Historia de una policía militarizada”, Ibero-Americana-Nordic Journal of Latin American Studies, Vol. XX, № 3, pp. 3-31.

Reiner, Robert; Bowling, Benjamin y ShePTyCKI, James (2019). The politics of the police (5 ed., Oxford University Press).

Rodríguez, Álvaro; Peña, Sebastián; Cavieres, Isabel; Vergara, María José; PÉrez, Marcela; Campos, Miguel; Peredo, Daniel; Jorquera, Patricio; Palma, Rodrigo; Cortés, Dennis; LóPEZ, Mauricio y Morales, Sergio (2020). "Ocular trauma by kinetic impact projectiles during civil unrest in Chile”, Eye, Vol. 35, pp. 1666-1672.

Simonson, Jocelyn (2021). "Police Reform through a Power Lens", Yale Law Journal, Vol. 130, $\mathrm{N}^{\circ} 4$, pp. 778-860.

Sklansky, David (2005). "Police and Democracy", Michigan Law Review, Vol. 103, Issue 7, pp. 1699-1830.

UnIVERSIDAD DE ChILE (2020): "Revista Eye de Nature publica y destaca investigación de la U. de Chile sobre daño ocular durante el estallido social”, Fuentes, Cristian, 25 de agosto de 2020, en: https://www.uchile.cl/noticias/166739/nature-destaca-investigacion-sobre-danoocular-tras-estallido-social.

ZEDNer, Lucia (2006). "Policing before and after the Police. The Historical Antecedents of Contemporary Crime Control”, British Journal of Criminology, Vol. 46, № 1, pp. 78-96.

ZEDNER, Lucia (2009). Security (Routledge). 


\section{NORMAS CITADAS}

\section{Argentina}

Constitución de la Nación Argentina de 22 de agosto de 1994.

\section{Alemania}

Ley Fundamental Alemana de 23 de mayo de 1949.

\section{Austria}

Constitución de Austria de 1 de octubre de 1920.

\section{Bélgica}

Constitución de Bélgica de 7 de febrero de 1831.

\section{Bolivia}

Constitución Política del Estado Plurinacional de Bolivia de 7 de febrero de 2009.

\section{Bulgaria}

Constitución de Bulgaria de 12 de julio de 1991.

\section{Brasil}

Constitución de la República Federativa del Brasil de 5 de octubre de 1988.

\section{Chile}

Decreto con Fuerza de Ley № 2484 de 5 de mayo de 1927 del Ministerio del Interior.

Decreto № 2.226 de 19 de diciembre de 1944 del Ministerio de Justicia. Código de Justicia Militar.

Decreto con Fuerza de Ley № 213 de 30 de marzo de 1960 del Ministerio de Hacienda. Aprueba la Ley Orgánica de Carabineros de Chile.

Ley № 17.398 de 9 de enero de 1971. Modifica la Constitución Política del Estado.

Decreto Ley № 444 de 27 de abril de 1974 del Ministerio del Interior. Incorpora y crea Subsecretaría de Carabineros en el Ministerio de Defensa Nacional.

Decreto Ley № 1063 de 9 de junio de 1975 del Ministerio de Defensa Nacional. Aprueba Ley Orgánica de Carabineros de Chile.

Constitución Política de la República de Chile de 21 de octubre de 1980.

Ley № 18.825 de 15 de junio de 1989 del Ministerio del Interior. Modifica la Constitución Política de la República de Chile.

Ley № 18.961 de 27 de febrero de 1990 del Ministerio de Defensa Nacional. Ley Orgánica Constitucional de Carabineros.

Ley № 20.050 de 18 de agosto de 2005 del Ministerio Secretaría General de la Presidencia. Reforma constitucional que introduce diversas modificaciones a la Constitución Política de la República. 
Ley № 20.477 de 10 de diciembre de 2010 del Ministerio de Defensa Nacional. Modifica competencia de Tribunales Militares.

Ley № 20.968 de 11 de noviembre de 2016 del Ministerio de Justicia y Derechos Humanos. Tipifica delitos de tortura y tratos crueles, inhumanos y degradantes.

\section{Chipre}

Constitución de Chipre de 6 de abril de 1960.

\section{Colombia}

Constitución Política de La República De Colombia de 4 de julio de 1991.

\section{Costa Rica}

Constitución Política de Costa Rica de 8 de noviembre de 1949.

\section{Croacia}

Constitución de la República de Croacia de 22 de diciembre de 1990.

\section{Cuba}

Constitución de la República de Cuba de 24 de febrero de 2019.

\section{Dinamarca}

Ley Constitucional de Dinamarca de 5 De junio de 1953.

\section{Ecuador}

Constitución de la República del Ecuador de 28 de septiembre de 2008.

\section{Estonia}

Constitución de Estonia de 28 de junio de 1992.

\section{El Salvador}

Constitución de la República de El Salvador de 15 de diciembre de 1983.

\section{España}

Constitución Española de 27 de diciembre de 1978.

\section{Eslovaquia}

Constitución de Eslovaquia de 1 de septiembre de 1992.

\section{Eslovenia}

Constitución de la República de Eslovenia de 23 de diciembre de 1991.

\section{Finlandia}


Constitución de Finlandia de 11 de junio de 1999.

\section{Francia}

Constitución Francesa de 4 de octubre de 1958.

\section{Grecia}

Constitución de Grecia de 9 de junio de 1975.

\section{Guatemala}

Constitución Política de la República de Guatemala de 31 de mayo de 1985.

\section{Honduras}

Constitución Política de Honduras de 11 de enero de 1982.

\section{Hungría}

Constitución de Hungría de 18 de abril de 2011.

\section{Irlanda}

Constitución de Irlanda de 29 de diciembre de 1937.

Italia

Constitución de la República italiana de 21 de diciembre de 1947.

\section{Letonia}

Constitución de Letonia de 15 de febrero de 1922.

\section{Lituania}

Constitución de Lituania de 25 de octubre de 1992.

\section{Luxemburgo}

Constitución de Luxemburgo de 17 de octubre de 1868.

\section{Malta}

Constitución de Malta de 21 de septiembre de 1964.

\section{México}

Constitución Política de los Estados Unidos Mexicanos de 5 de febrero de 1917.

\section{Nicaragua}

Constitución Política de la República de Nicaragua de 9 de enero de 1987. 


\section{Países Bajos}

Constitución de los Países Bajos de 29 de marzo de 1814.

\section{Panamá}

Constitución Política de la República de Panamá de 11 de octubre de 1972.

\section{Perú}

Constitución Política del Perú de 29 de diciembre de 1993.

\section{Polonia}

Constitución de Polonia de 2 de abril de 1997.

\section{Portugal}

Constitución de Portugal de 25 de abril de 1976.

\section{República Checa}

Constitución de la República Checa de 16 de diciembre de 1992.

\section{República Dominicana}

Constitución de la República Dominicana de 14 de junio del 2015.

\section{Rumania}

Constitución de Rumania de 21 de noviembre de 1991.

\section{Suiza}

Constitución Federal de la Confederación Suiza de 18 de abril de 1999.

\section{Uruguay}

Constitución de la República Oriental del Uruguay de 15 de febrero de 1967.

\section{Venezuela}

Constitución de la República Bolivariana de Venezuela de 15 de diciembre de 1999. 